\title{
GLULAM BEAMS WITH HOLES
}

\author{
Mario Jeleč \\ Josip Juraj Strossmayer University of Osijek, Faculty of Civil Engineering Osijek, mag.ing.aedif. \\ Damir Varevac \\ Josip Juraj Strossmayer University of Osijek, Faculty of Civil Engineering Osijek, Associate Professor \\ Corresponding author: dvarevac@gfos.hr \\ Jurko Zovkić \\ Josip Juraj Strossmayer University of Osijek, Faculty of Civil Engineering Osijek, Assistant Professor
}

\begin{abstract}
This paper provides a systematic review of research on glued laminated timber beams with circular and rectangular openings. Experiments on girders with unreinforced openings varied several parameters, including the girder span and shape, opening position and shape, and the relationship between the stress state near the opening and the ratio of opening size to girder size. We compare experimental results with recommendations given by DIN 1052:2004-08, DIN EN 1995-1-1/NA: 2010, prEN 1995-1-1: Final Draft, analytical expressions, and other relevant standards, as well as with the results of numerical models using the finite element method. Because of its myriad complexities and uncertainties, this area remains open for further research and for implementation of that research into practical design guidelines and rules.
\end{abstract}

Keywords: glulam beams, rectangular openings, circular openings, stress distribution

\section{LIJEPLJENI LAMELIRANI NOSAČI S OTVORIMA}

Sažetak: U radu je prikazan sustavan pregled dostupnih istraživanja o lijepljenim lameliranim nosačima s kružnim i pravokutnim otvorima. Prikazani su rezultati ispitivanja na nosačima bez ojačanja otvora, pri čemu je varirano nekoliko utjecajnih parametara kao što su raspon i poprečni presjek nosača, položaj i oblik otvora, odnos između veličine otvora $i$ veličine nosača te stanje naprezanja na mjestu otvora nosača. Preuzeti rezultati eksperimentalnih ispitivanja iz literature su uspoređeni s preporukama i analitičkim izrazima prema DIN 1052:2004-08, DIN EN 1995-1-1/NA: 2010, prEN1995-1-1: Final Draft i drugim važnijim normama, te isto tako is rezultatima numeričkih analiza modeliranih metodom konačnih elemenata. S obzirom da europska norma za drvene konstrukcije, EN 1995-1-1:2004, ne sadrži postupak dimenzioniranja LLD nosača s otvorima, iako neki nacionalni dodaci daju svoje preporuke, ovo područje ostaje otvoreno za daljnja istraživanja i njihovu implementaciju u postupak dimenzioniranja, primjenjiv u svakodnevnoj inženjerskoj uporabi.

Ključne riječi: lijepljeni lamelirani nosači, pravokutni otvori, kružni otvori, raspodjela naprezanja 


\section{INTRODUCTION}

Placing openings in main and secondary roof and ceiling beams is a common practice because of engineering, installation, or architectural requirements. Introducing openings in beam girders changes the stress state and significantly weakens the cross-section. This problem is emphasized in beams made of anisotropic materials such as glued laminated timber (Figure 1). Moreover, such beams experience alternate tensile and compressive stresses perpendicular to the fiber direction, which hampers bearing capacity. Since the wood has a low shear and extremely low tensile strength perpendicular to the fibers and that collapse due to reaching these strengths is brittle, these beams should be carefully designed and openings carefully considered because each new one further endangers the safety of the structure. Similar stress state occurs even in curved beams and end-notched beams, but their analysis is not included in this paper.

Because glued laminated (glulam) beams with openings are complicated and can have many issues, their design is treated differently in various international standards; notably, the European structural code EN 1995-1-1 [2] does not provide any design provisions for these beams.
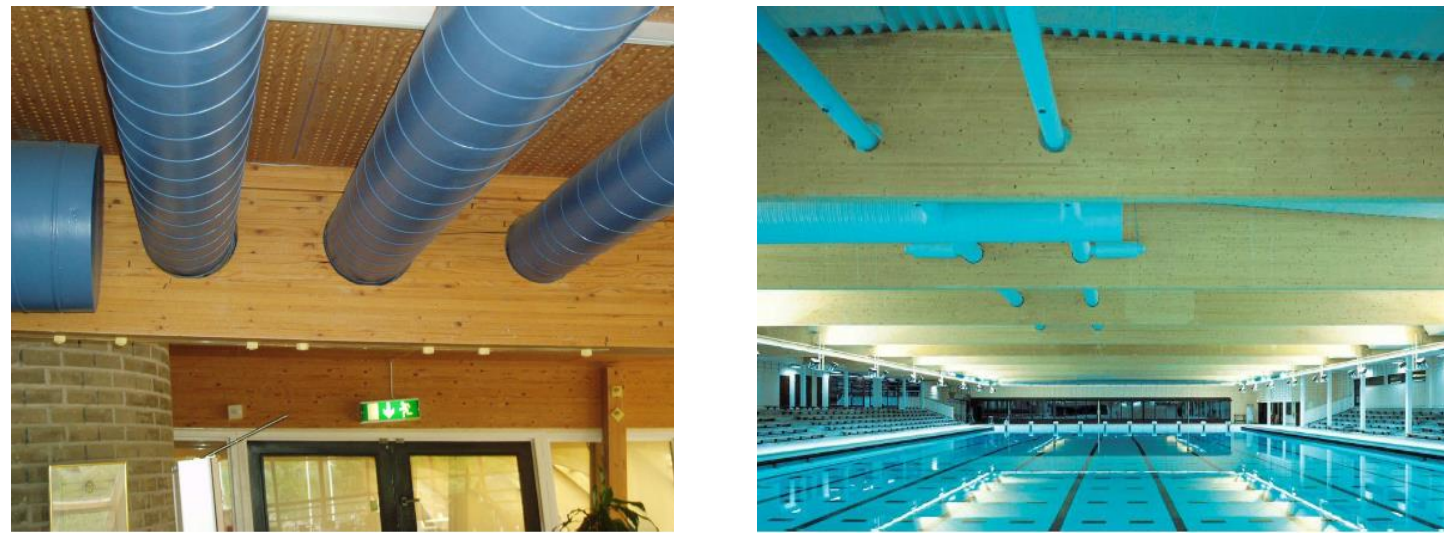

Figure 1 Glulam beams with openings [1]

\section{STRESS STATES NEAR OPENINGS}

Openings in glulam beams, whether in the pure bending zone or the bending-shear interaction zone, drastically change the stress state, which disturbs the flow of normal and shear stresses, concentrating tensile and compressive stresses perpendicular to the fiber direction. Figure 2 shows examples of stress distributions around circular and rectangular openings. For openings closer to the girder support, where bending and shear stresses interact, it is common for tensile stresses perpendicular to the fibers to appear on two diagonally opposite edges of the opening; in contrast, for pure bending, tensile stresses appear on the two upper edges of the opening. The tensile stress decreases exponentially with distance from the edge of the opening (Figure 3 ). The position of the peak tensile stress mostly depends on the stress state, the ratio of the bending moment and shear force $(M / V)$, the ratio of opening diameter and height of beam $(\mathrm{d} / \mathrm{h})$, and the position of the opening relative to the neutral axis of beam [3]. The maximum tensile stress usually occurs at $60^{\circ}$ relative to the beam axis for circular openings and pure bending [4], at $45^{\circ}$ for interacting shear and bending [5], and at $40^{\circ}$ for pure shear [6]. Aicher and Höfflin [7] found that cracks appeared in similar locations for rectangular and circular openings, but the sharp corners of the rectangular opening decreased its bearing capacity, leading to earlier collapse. They also studied how the opening dimensions affected the maximum tensile stress perpendicular to the grain, concluding that square openings are the worst because they cracked earlier and had lower maximum stresses than the rectangular openings. Because glulam beams with openings are mainly used as roofs and ceiling girders, which are subjected to bending, they may experience superimposed normal stresses parallel to the beam axis, which must also be considered. Aicher and Höfflin [8] also investigated how longitudinal compressive forces interact with openings, finding that these stresses are unfavorable because they concentrate tensile stresses around the openings, just as was described for moments and shear forces. 


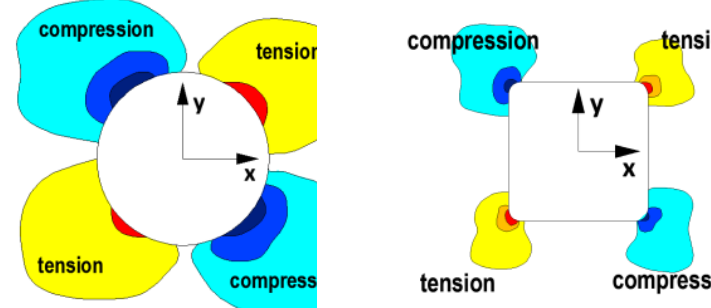

(a)
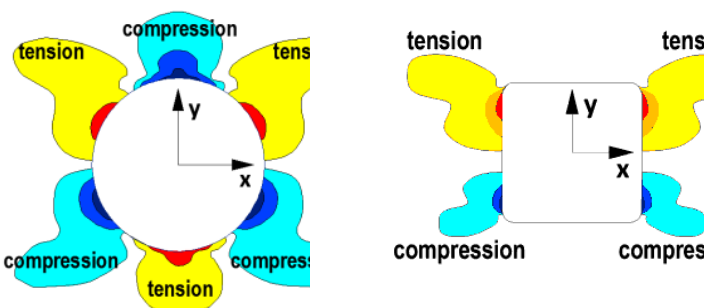

(b)

Figure 2 Distribution of compressive and tensile stresses perpendicular to the grain, around circular and rectangular openings, for these stress states:(a)interaction of shear and bending;(b)pure bending $[6,7,9]$

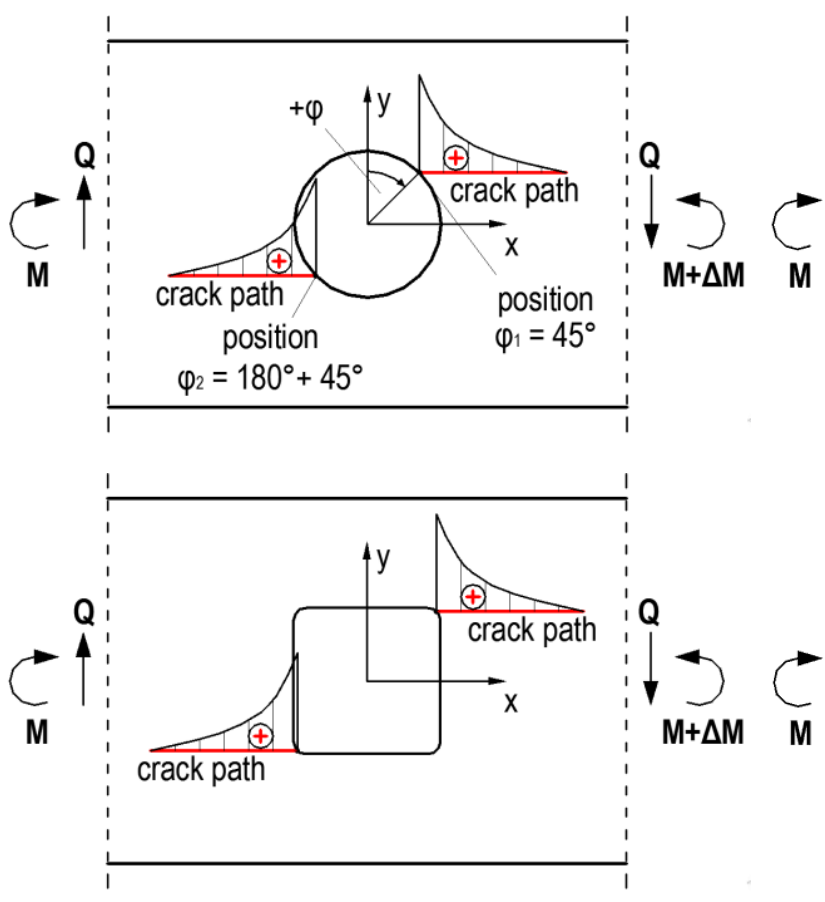

(a)
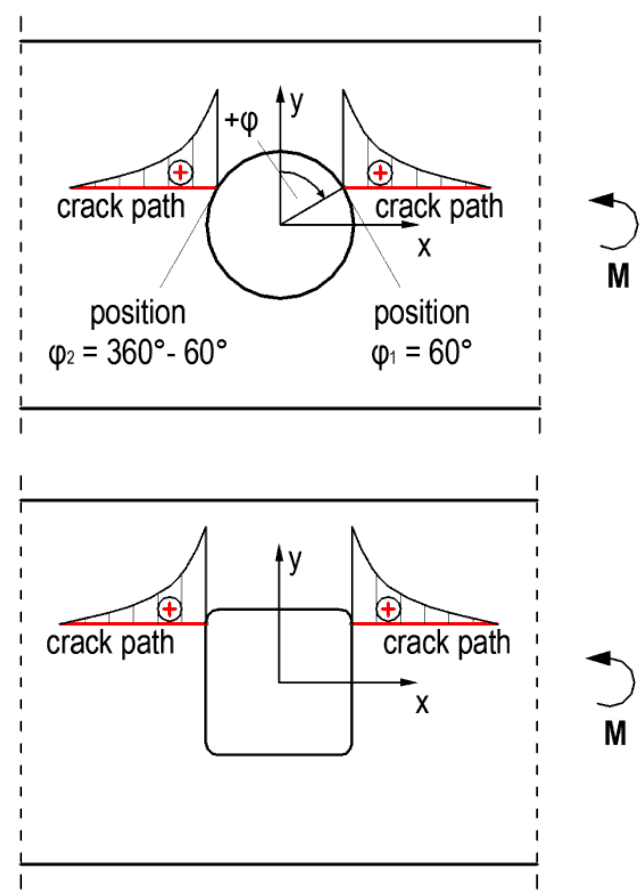

(b)

Figure 3 Locations of cracks for circular and rectangular openings for these stress states: (a) interaction of shear and bending; (b) pure bending $[3,5]$

\section{EXPERIMENTAL RESULTS}

Glulam beams with openings were first tested in 1971 by Bengtsson and Dahl [10]. Using three-point bending, they studied 9 beams with spans of $5 \mathrm{~m}$ and circular and rectangular openings near the support, in the shearbending interaction zone. In most of these tests, cracks appeared at loads of $70-90 \%$ of the failure loads. They found that the beams could be strengthened by adding 10 -mm-thick plywood boards, glue-nailed on both sides of the beams. In 1977, Kolb and Frech [11] tested 12 beams with spans of $8 \mathrm{~m}$ with four-point bending, placing the openings in a region dominated by shear and in one with a pure bending moment. They found that, for beams with an opening in the region with a pure moment, their capacities were limited by bending failure at the midspan. In 1980, Penttala [12] tested 6 beams with spans of $4 \mathrm{~m}$ and 4 beams with spans of $5 \mathrm{~m}$, placing circular and rectangular openings in a region subject to both shear force and a bending moment, while varying the dimensions and position of the openings. In 1983, Johannesson [13] performed a comprehensive study of 45 beams with various cross-sections, spans, girders shapes, and opening positions. He also investigated how long-term load (2 months, concentrated force of $30 \mathrm{kN}$ ) affected these beams. In 1991, Pizio [14] tested glulam beams with spans up to $2 \mathrm{~m}$ with rectangular openings with sharp corners. Some of these beams were reinforced with bolts near the 
openings. In 1995, Hallstrom [15] tested reinforced and unreinforced openings in glulam beams with spans up to $6 \mathrm{~m}$, finding some advantages to using glass fibers as reinforcement. In 2005, Höfflin [16] made a major research contribution by testing 68 glulam beams with unreinforced circular openings, varying the load configuration, span, cross-sectional dimensions of beams, and diameters and locations of the openings. A year later, Aicher and Höfflin [17] tested 15 straight beams with maximum spans of $9.5 \mathrm{~m}$ and, for the first time, 6 curved beams, each with a radius of curvature of 15 or $30 \mathrm{~m}$. In 2008, Danielsson $[18,19,20]$ examined rectangular openings on 36 samples, varying four influential parameters: beam height, stress state (ratio of bending moment and shear force), material strength, as well as the positions of the openings relative to the beam height, which had not previously been studied (Figure 4).

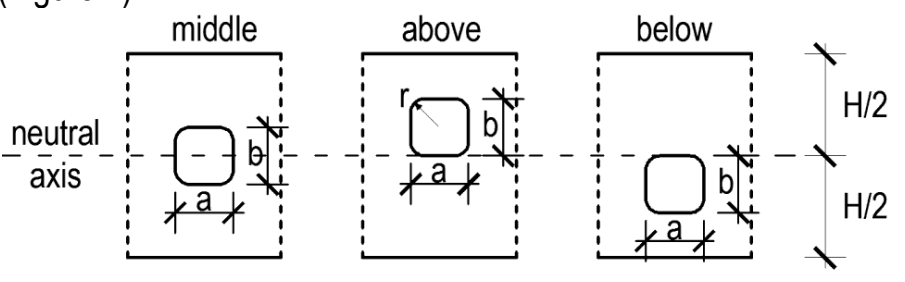

Figure 4 Dimensions for eccentrically positioned openings [18]

Aicher and Höfflin's results showed that the beam size greatly affected capacity: increasing the beam size by 3.5 times, while keeping constant the width and ratio between the opening dimensions and beam height, decreased the capacity up to $35 \%$. By increasing the beam dimensions the probability of faults in it (slope of the fibers, knots and other irregularities) is also increased, but also cross section becomes more slender which causes stability problems in the area of the large bending stresses. Such a result also indicates questionable reliability of the tests performed on a scale model and emphasizes the importance of performing tests on full size beams. They also found that beams with eccentrically positioned openings, relative to the neutral axis of the beam, had capacities up to $15 \%$ lower than those with centrically placed openings. Moreover, they found that material strength negligibly affected the performance of homogeneous and combined glulam beams. Finally, they found that beams with openings positioned in the pure shear zone had higher capacity, up to $10 \%$ higher than those with openings positioned in the bending-shear interaction zone.

Figures 5 and 6 show the test setups used in previous reports, and Figure 7 shows the crack locations for various test setups and stress states. Table 1 shows the results of tests on circular openings, and Table 2 shows those for rectangular openings in the bending-shear interaction zone. These tables show the most important parameters, including the test models, cross-sections, shapes and dimensions of the openings, ratio between bending and shear, number of tested samples, locations of cracks, and three characteristic values of transverse forces: $V_{c, 0}, V_{c}$, and $V_{f} . V_{c, 0}$ is the shear force at the center of the opening when the crack forms, $V_{c}$ is the shear force when the crack has propagated over the entire beam width, and $V_{f}$ is the shear force at beam failure (Figure 8).

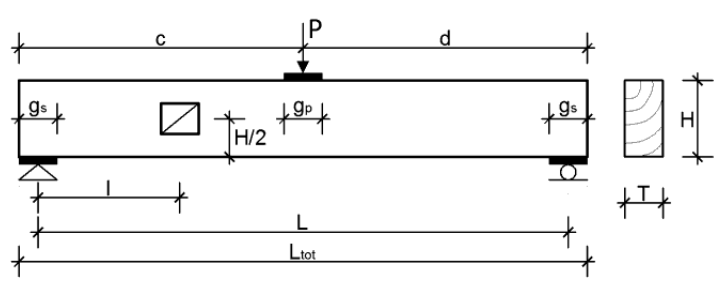

(A)

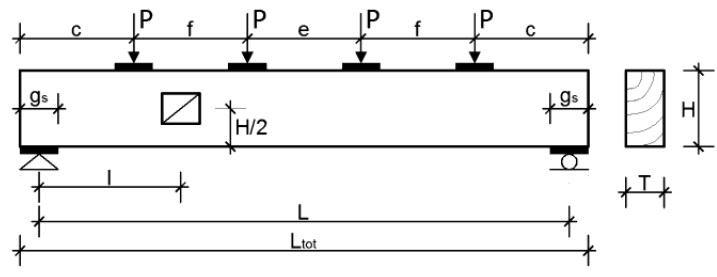

(C)

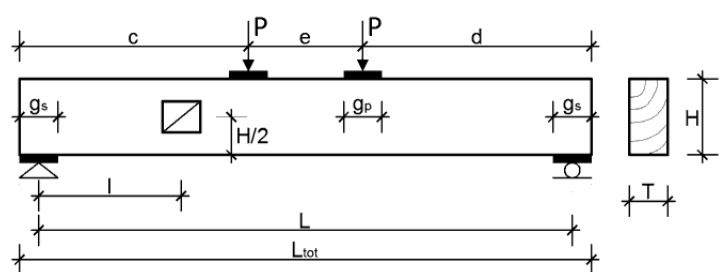

(B)

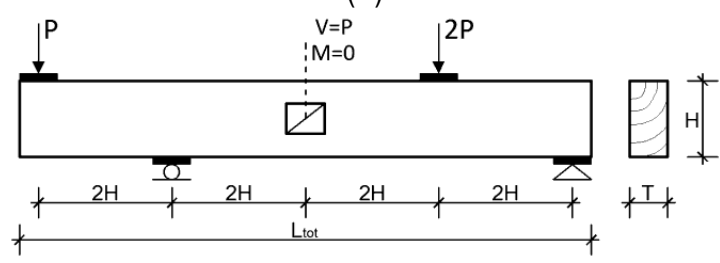

(D)

Figure 5 Test setups for straight beams $[1,20]$ 


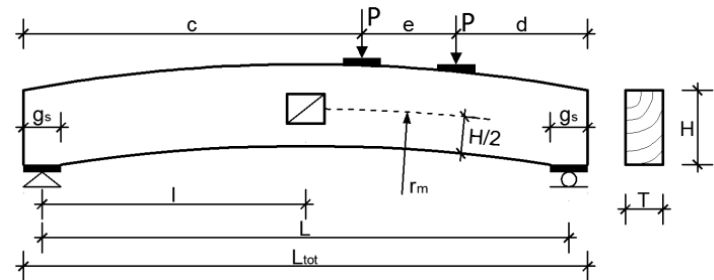

(E)

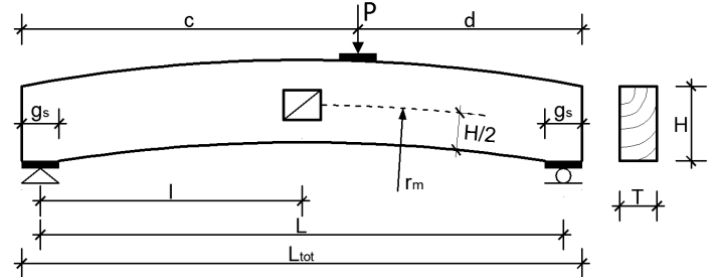

(F)

Figure 6 Test setups for curved beams (E and F) [1]

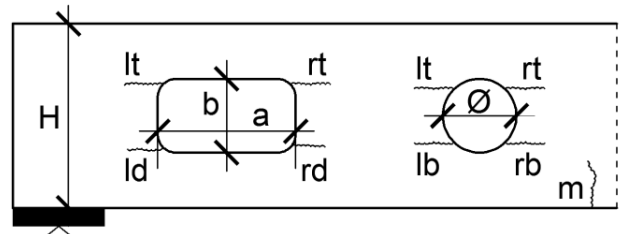

Figure 7 Opening dimensions and crack locations [1]

Table 1 Results for beams with circular openings in regions dominated by shear force

\begin{tabular}{|c|c|c|c|c|c|c|c|c|c|c|c|c|}
\hline \multirow{2}{*}{$\begin{array}{l}\text { Researcher } \\
\text { (year) }\end{array}$} & \multirow{2}{*}{$\begin{array}{l}\text { Test } \\
\text { setup }\end{array}$} & \multirow{2}{*}{$\begin{array}{l}T \times H \\
{[\mathrm{~mm}]}\end{array}$} & \multirow{2}{*}{$\begin{array}{c}\Phi \\
{[\mathrm{mm}]}\end{array}$} & \multirow{2}{*}{$\begin{array}{c}\frac{M}{V H} \\
{[-]}\end{array}$} & \multirow[b]{2}{*}{$\mathrm{n}$} & \multicolumn{2}{|c|}{$V_{c, 0}$} & \multicolumn{2}{|c|}{$V_{c}$} & \multicolumn{2}{|c|}{$V_{f}$} & \multirow[b]{2}{*}{ LoC } \\
\hline & & & & & & $\begin{array}{c}\text { mean } \\
{[\mathrm{kN}]}\end{array}$ & $\begin{array}{l}\text { std } \\
{[\mathrm{kN}]}\end{array}$ & $\begin{array}{c}\text { mean } \\
{[\mathrm{kN}]}\end{array}$ & $\begin{array}{l}\text { std } \\
{[\mathrm{kN}]}\end{array}$ & $\begin{array}{c}\text { mean } \\
{[\mathrm{kN}]}\end{array}$ & $\begin{array}{l}\text { std } \\
{[\mathrm{kN}]}\end{array}$ & \\
\hline $\begin{array}{l}\text { Bengtsson } \\
\text { and Dahl } \\
\text { (1970) [10] }\end{array}$ & $\begin{array}{l}A \\
A\end{array}$ & $\begin{array}{l}90 \times 500 \\
90 \times 500\end{array}$ & $\begin{array}{l}250 \\
150\end{array}$ & $\begin{array}{l}1.20 \\
1.20\end{array}$ & $\begin{array}{l}2 \\
1\end{array}$ & & & & & $\begin{array}{l}38.4 \\
52.5\end{array}$ & 1.2 & $\begin{array}{c}\mathrm{Ib}, \mathrm{rt} \\
\mathrm{m}\end{array}$ \\
\hline $\begin{array}{l}\text { Penttala } \\
\text { (1980) [12] }\end{array}$ & $\begin{array}{l}A \\
A \\
A \\
A \\
A\end{array}$ & $\begin{array}{c}90 \times 500 \\
90 \times 500 \\
90 \times 500 \\
115 \times 800 \\
115 \times 800\end{array}$ & $\begin{array}{l}255 \\
250 \\
150 \\
400 \\
300\end{array}$ & $\begin{array}{l}1.20 \\
2.10 \\
1.20 \\
1.03 \\
2.00\end{array}$ & \begin{tabular}{l|}
1 \\
1 \\
1 \\
1 \\
1
\end{tabular} & 57.1 & & & & $\begin{array}{l}33.8 \\
31.6 \\
51.3 \\
65.9 \\
89.5\end{array}$ & & $\begin{array}{l}\text { lb, rt } \\
\text { lb, rt } \\
\text { lb, rt } \\
\text { lb, rt } \\
\text { lb, rt }\end{array}$ \\
\hline $\begin{array}{l}\text { Johannesson } \\
\text { (1983) [13] }\end{array}$ & $\begin{array}{l}A \\
A \\
A \\
A \\
A \\
A\end{array}$ & $\begin{array}{l}90 \times 500 \\
90 \times 500 \\
90 \times 500 \\
90 \times 500 \\
88 \times 495 \\
88 \times 495\end{array}$ & $\begin{array}{l}250 \\
250 \\
250 \\
125 \\
125 \\
396 \\
\end{array}$ & $\begin{array}{l}1.30 \\
2.80 \\
0.60 \\
0.60 \\
2.53 \\
2.53 \\
\end{array}$ & \begin{tabular}{l|}
2 \\
2 \\
2 \\
2 \\
4 \\
4
\end{tabular} & & & $\begin{array}{l}29.6 \\
33.2 \\
33.8 \\
\\
\\
51.9 \\
16.1 \\
\end{array}$ & $\begin{array}{l}5.4 \\
2.6 \\
7.1 \\
\\
4.6 \\
1.5 \\
\end{array}$ & $\begin{array}{l}36.5 \\
37.5 \\
41.7 \\
40.1\end{array}$ & $\begin{array}{l}4.3 \\
3.5 \\
4.1 \\
0.1\end{array}$ & $\begin{array}{l}\mathrm{lb}, \mathrm{rt} \\
\mathrm{lb}, \mathrm{rt} \\
\mathrm{lb}, \mathrm{rt} \\
\mathrm{lb}, \mathrm{rt} \\
\mathrm{rt} \\
\mathrm{lb}, \mathrm{rt}\end{array}$ \\
\hline $\begin{array}{l}\text { Hallström } \\
\text { (1995) [15] }\end{array}$ & $A$ & $90 \times 315$ & 150 & 2.78 & 5 & & & 24.5 & 3.5 & & & $\mathrm{lb}, \mathrm{rt}$ \\
\hline $\begin{array}{l}\text { Höfflin } \\
(2005) \text { [16] }\end{array}$ & $\begin{array}{l}\text { B } \\
B \\
B \\
C \\
A \\
A \\
A \\
C\end{array}$ & $\begin{array}{l}120 \times 900 \\
120 \times 900 \\
120 \times 900 \\
120 \times 900 \\
120 \times 450 \\
120 \times 450 \\
120 \times 450 \\
120 \times 450\end{array}$ & $\begin{array}{c}180 \\
270 \\
360 \\
270 \\
90 \\
135 \\
180 \\
135\end{array}$ & $\begin{array}{l}1.50 \\
1.50 \\
1.50 \\
5.00 \\
1.50 \\
1.50 \\
1.50 \\
5.00\end{array}$ & \begin{tabular}{l|}
5 \\
6 \\
6 \\
5 \\
5 \\
6 \\
4 \\
5
\end{tabular} & $\begin{array}{l}69.2 \\
65.3 \\
57.6 \\
43.1 \\
62.8 \\
47.4 \\
34.6 \\
34.7\end{array}$ & $\begin{array}{c}23.2 \\
22.1 \\
16.8 \\
8.3 \\
15.6 \\
14.2 \\
7.4 \\
18.2\end{array}$ & $\begin{array}{l}106.4 \\
96.4 \\
69.2 \\
55.1 \\
76.8 \\
65.5 \\
47.6 \\
58.0\end{array}$ & $\begin{array}{c}27.8 \\
11.7 \\
9.0 \\
8.6 \\
13.8 \\
7.6 \\
8.5 \\
7.1\end{array}$ & $\begin{array}{c}128.1 \\
108.7 \\
87.5 \\
84.2 \\
82.1 \\
67.9 \\
51.8 \\
63.4\end{array}$ & $\begin{array}{c}19.2 \\
6.7 \\
15.6 \\
18.0 \\
7.6 \\
7.0 \\
5.9 \\
6.5\end{array}$ & $\begin{array}{l}\mathrm{lb}, \mathrm{rt} \\
\mathrm{lb}, \mathrm{rt} \\
\mathrm{lb}, \mathrm{rt} \\
\mathrm{lb}, \mathrm{rt} \\
\mathrm{lb}, \mathrm{rt} \\
\mathrm{lb}, \mathrm{rt} \\
\mathrm{lb}, \mathrm{rt} \\
\mathrm{lb}, \mathrm{rt}\end{array}$ \\
\hline $\begin{array}{l}\text { Aicher and } \\
\text { Höfflin } \\
\text { (2006) [17] }\end{array}$ & $\begin{array}{l}C \\
C \\
C \\
E \\
F\end{array}$ & $\begin{array}{c}120 \times 900 \\
120 \times 900 \\
120 \times 450 \\
120 \times 450^{1)} \\
120 \times 900^{1)}\end{array}$ & $\begin{array}{l}180 \\
360 \\
180 \\
180 \\
360\end{array}$ & $\begin{array}{l}5.00 \\
5.00 \\
5.00 \\
5.00 \\
5.00\end{array}$ & $\begin{array}{l}4 \\
5 \\
6 \\
3 \\
3\end{array}$ & $\begin{array}{l}66.4 \\
46.7 \\
42.4 \\
15.4 \\
33.5\end{array}$ & $\begin{array}{c}21.5 \\
15.3 \\
9.6 \\
3.1 \\
13.6\end{array}$ & $\begin{array}{l}106.4 \\
61.6 \\
48.8 \\
37.9 \\
49.6\end{array}$ & $\begin{array}{c}15.0 \\
15.0 \\
7.7 \\
6.8 \\
17.4\end{array}$ & $\begin{array}{l}111.6 \\
79.9 \\
53.7 \\
44.8 \\
66.6\end{array}$ & $\begin{array}{l}13.1 \\
3.2 \\
8.0 \\
2.5 \\
6.9\end{array}$ & $\begin{array}{l}\mathrm{rt} \\
\mathrm{lb}, \mathrm{rt} \\
\mathrm{lb}, \mathrm{rt} \\
\mathrm{lb}, \mathrm{rt} \\
\mathrm{lb}, \mathrm{rt}\end{array}$ \\
\hline $\begin{array}{l}T \text { - width of } \\
H \text { - height of } \\
n-\text { number } 0 \\
\text { LoC - locatio } \\
\text { 1) - curved be }\end{array}$ & secti & $\begin{array}{l}\text { d } \\
\text { acronym } \\
03\end{array}$ & III & $\mathrm{r}$ & & & & & & & & \\
\hline
\end{tabular}


Table 2 Results for beams with rectangular openings in regions dominated by shear force

\begin{tabular}{|c|c|c|c|c|c|c|c|c|c|c|c|c|c|}
\hline \multirow{2}{*}{$\begin{array}{l}\text { Researcher } \\
\text { (year) }\end{array}$} & \multirow[b]{2}{*}{ TS } & \multirow{2}{*}{$\begin{array}{c}T \times H \\
{[\mathrm{~mm}]}\end{array}$} & \multirow{2}{*}{$\begin{array}{c}a \times b \\
{[\mathrm{~mm}]}\end{array}$} & \multirow[b]{2}{*}{$r$} & \multirow{2}{*}{$\begin{array}{c}\frac{M}{V H} \\
{[-]} \\
\end{array}$} & \multirow[b]{2}{*}{$n$} & \multicolumn{2}{|c|}{$V_{c 0}$} & \multicolumn{2}{|c|}{$V_{c}$} & \multicolumn{2}{|c|}{$V_{f}$} & \multirow[b]{2}{*}{ LoC } \\
\hline & & & & & & & $\begin{array}{r}\text { mean } \\
{[\mathrm{kN}]}\end{array}$ & $\begin{array}{r}\text { std } \\
{[\mathrm{kN}]} \\
\end{array}$ & $\begin{array}{r}\text { mean } \\
{[\mathrm{kN}]} \\
\end{array}$ & $\begin{array}{l}\text { std } \\
{[\mathrm{kN}]}\end{array}$ & $\begin{array}{l}\text { mean } \\
{[\mathrm{kN}]} \\
\end{array}$ & $\begin{array}{l}\text { std } \\
{[\mathrm{kN}]}\end{array}$ & \\
\hline \multirow{2}{*}{$\begin{array}{l}\text { Bengtsson } \\
\text { and Dahl } \\
(1970)[10]\end{array}$} & $A$ & $90 \times 500$ & $300 \times 150$ & 0 & 1.20 & 2 & & & & & 39.0 & 0.3 & $\mathrm{lb}, \mathrm{rt}$ \\
\hline & $A$ & $90 \times 500$ & $200 \times 100$ & 0 & 1.20 & 2 & & & & & 49.6 & 1.1 & $\mathrm{lb}, \mathrm{rt}$ \\
\hline \multirow{4}{*}{$\begin{array}{l}\text { Kolb and } \\
\text { Frech } \\
\text { (1977) [11] }\end{array}$} & $B$ & $80 \times 550$ & $250 \times 250$ & $?$ & 0.91 & 2 & & & & & 32.7 & 2.1 & $\mathrm{Ib}, \mathrm{rt}$ \\
\hline & B & $80 \times 550$ & $250 \times 150$ & $?$ & 0.91 & 2 & & & & & 44.0 & 2.8 & $\mathrm{lb}, \mathrm{rt}$ \\
\hline & B & $80 \times 550$ & $250 \times 250$ & $?$ & 1.82 & 2 & & & & & 33.8 & 1.1 & $\mathrm{lb}, \mathrm{rt}$ \\
\hline & $\mathrm{B}$ & $80 \times 550$ & $250 \times 150$ & $?$ & 1.82 & 2 & & & & & 35.4 & 4.0 & $\mathrm{lb}, \mathrm{rt}$ \\
\hline \multirow{5}{*}{$\begin{array}{l}\text { Penttala } \\
\text { (1980) [12] }\end{array}$} & $A$ & $90 \times 500$ & $200 \times 200$ & $?$ & 1.60 & 1 & & & & & 33.8 & & $\mathrm{Ib}, \mathrm{rt}$ \\
\hline & $A$ & $90 \times 500$ & $400 \times 200$ & $?$ & 1.60 & 1 & 25.0 & & & & 31.3 & & $\mathrm{lb}, \mathrm{rt}$ \\
\hline & $A$ & $90 \times 500$ & $600 \times 200$ & $?$ & 1.60 & 1 & 20.8 & & & & 30.0 & & $\mathrm{lb}, \mathrm{rt}$ \\
\hline & $A$ & $115 \times 800$ & $400 \times 200$ & $?$ & 1.25 & 1 & & & & & 69.1 & & $\mathrm{lb}, \mathrm{rt}$ \\
\hline & A & $115 \times 800$ & $200 \times 200$ & $?$ & 1.25 & 1 & 52.5 & & & & 84.4 & & $\mathrm{lb}, \mathrm{rt}$ \\
\hline \multirow{8}{*}{$\begin{array}{l}\text { Johannesson } \\
\text { (1983) [13] }\end{array}$} & $A$ & $90 \times 500$ & $250 \times 250$ & 25 & 1.30 & 2 & & & 26.8 & 0.5 & 28.5 & 2.8 & $\mathrm{Ib}, \mathrm{rt}$ \\
\hline & $A$ & $90 \times 500$ & $250 \times 250$ & 25 & 2.80 & 2 & & & 22.2 & 2.3 & 25.6 & 0.6 & $\mathrm{lb}, \mathrm{rt}$ \\
\hline & $A$ & $140 \times 400$ & $600 \times 200$ & 25 & 2.25 & 1 & & & 30.0 & & 37.0 & & $\mathrm{lb}$ \\
\hline & $A$ & $88 \times 495$ & $125 \times 125$ & 25 & 2.53 & 4 & & & 40.4 & 11.1 & & & $\mathrm{lb}, \mathrm{rt}$ \\
\hline & $A$ & $88 \times 495$ & $375 \times 125$ & 25 & 2.53 & 4 & & & 37.7 & 6.4 & & & $\mathrm{rt}$ \\
\hline & $A$ & $88 \times 495$ & $370 \times 370$ & 25 & 2.53 & 4 & & & 9.1 & 2.1 & & & $\mathrm{rt}$ \\
\hline & $A$ & $88 \times 495$ & $735 \times 245$ & 25 & 2.53 & 4 & & & 12.8 & 1.1 & & & $\mathrm{lb}, \mathrm{rt}$ \\
\hline & $A$ & $88 \times 495$ & $1100 \times 370$ & 25 & 2.53 & 4 & & & 4.2 & 0.3 & & & $\mathrm{rt}$ \\
\hline \multirow{11}{*}{$\begin{array}{l}\text { Pizio } \\
(1991) \text { [14] }\end{array}$} & $A$ & $120 \times 400$ & $180 \times 180$ & 0 & 1.05 & 2 & 24.1 & 12.4 & 30.6 & 3.1 & 63.7 & 4.6 & $\mathrm{lb}, \mathrm{rt}$ \\
\hline & $A$ & $120 \times 400$ & $180 \times 90$ & 0 & 1.05 & 2 & 37.2 & 15.4 & 54.9 & 3.4 & 75.5 & 1.6 & $\mathrm{rt}$ \\
\hline & $A$ & $120 \times 400$ & $180 \times 10$ & 0 & 1.05 & 2 & 92.5 & 26.3 & 103 & 14.8 & 103 & 14.8 & $\mathrm{lb}, \mathrm{rt}$ \\
\hline & $A$ & $120 \times 400$ & $180 \times 90$ & 0 & 1.05 & 1 & 56.6 & & 71.0 & & 84.5 & & $\mathrm{lb}, \mathrm{rt}$ \\
\hline & $A$ & $120 \times 400$ & $180 \times 10$ & 0 & 1.05 & 1 & 110 & & 110 & & 110 & & $\mathrm{lb}, \mathrm{rt}$ \\
\hline & $A$ & $120 \times 400$ & $360 \times 180$ & 0 & 1.75 & 2 & 21.7 & 2.3 & 23 & 0.0 & 24.8 & 2.1 & $\mathrm{lb}, \mathrm{rt}$ \\
\hline & $A$ & $120 \times 400$ & $10 \times 180$ & 0 & 1.75 & 1 & 34.0 & & 34.0 & & 34.0 & & $\mathrm{lb}, \mathrm{rt}$ \\
\hline & $A$ & $120 \times 400$ & $360 \times 180$ & 0 & 1.75 & 1 & 19.2 & & 21.1 & & 28.8 & & $\mathrm{lb}, \mathrm{rt}$ \\
\hline & $A$ & $120 \times 400$ & $10 \times 180$ & 0 & 1.75 & 2 & 30.0 & 1.1 & 33.8 & 0.0 & 33.8 & 0.0 & $\mathrm{lb}, \mathrm{rt}$ \\
\hline & $A$ & $120 \times 400$ & $180 \times 90$ & 0 & 1.75 & 3 & 45.8 & 11.2 & 54.2 & 7.0 & 54.2 & 0.7 & $\mathrm{lb}, \mathrm{rt}$ \\
\hline & $A$ & $120 \times 400$ & $180 \times 180$ & 0 & 1.05 & 2 & 20.6 & 4.9 & 26.8 & 3.8 & 70.0 & 11.2 & $\mathrm{lb}, \mathrm{rt}$ \\
\hline \multirow{5}{*}{$\begin{array}{l}\text { Hallström } \\
\text { (1995) [15] }\end{array}$} & $A$ & $90 \times 315$ & $400 \times 150$ & 25 & 2.78 & 5 & & & 11.9 & 1.5 & & & $\mathrm{lb}, \mathrm{rt}$ \\
\hline & $A$ & $90 \times 315$ & $400 \times 150$ & 0 & 2.78 & 5 & & & 12.2 & 1.1 & & & $\mathrm{lb}, \mathrm{rt}$ \\
\hline & $A$ & $90 \times 315$ & $400 \times 150$ & 25 & 2.78 & 5 & & & 12.2 & 0.5 & & & $\mathrm{lb}, \mathrm{rt}$ \\
\hline & $A$ & $90 \times 315$ & $400 \times 150$ & 25 & $?$ & 1 & & & 12.2 & & & & $\mathrm{lb}, \mathrm{rt}$ \\
\hline & A & $165 \times 585$ & $600 \times 295$ & 25 & $?$ & 4 & & & 27.1 & 1.9 & & & $\mathrm{lb}, \mathrm{rt}$ \\
\hline \multirow{9}{*}{$\begin{array}{l}\text { Danielsson } \\
\text { (2008) [19] }\end{array}$} & $A^{S}$ & $115 \times 630$ & $210 \times 210$ & 25 & 2.0 & 4 & 44.8 & 3.9 & 57.3 & 8.1 & 60.6 & 8.0 & \\
\hline & $A^{S}{ }_{C}$ & $115 \times 630$ & $210 \times 210$ & 25 & 2.0 & 4 & 48.5 & 11.1 & 53.2 & 7.5 & 58.8 & 6.0 & \\
\hline & $A^{G}$ & $115 \times 630$ & $210 \times 210$ & 25 & 2.0 & 4 & 43.7 & 13.6 & 55.7 & 2.8 & 58.3 & 1.9 & \\
\hline & $A^{D}$ & $115 \times 630$ & $210 \times 210$ & 25 & 2.0 & 4 & 41.2 & 1.9 & 50.0 & 3.8 & 65.8 & 5.1 & \\
\hline & $D^{S}$ & $115 \times 630$ & $210 \times 210$ & 7 & 0.0 & 4 & 51.4 & 3.4 & 62.2 & 2.5 & & & \\
\hline & $A^{S}$ & $115 \times 180$ & $60 \times 60$ & 7 & 2.0 & 4 & 21.6 & 2.9 & 25.6 & 2.0 & 27.3 & 1.7 & \\
\hline & $A^{G}$ & $115 \times 180$ & $60 \times 60$ & 7 & 2.0 & 4 & 18.8 & 1.6 & 23.4 & 1.4 & 23.6 & 2.2 & \\
\hline & $A^{D}$ & $115 \times 180$ & $60 \times 60$ & 7 & 2.0 & 4 & 19.5 & 2.2 & 23.0 & 1.2 & 26.6 & 2.2 & \\
\hline & $D^{S}$ & $115 \times 180$ & $60 \times 60$ & 7 & 0.0 & 4 & 24.0 & 1.8 & 26.6 & 1.8 & & & \\
\hline
\end{tabular}

TS - test setup

$s$ - hole in the middle of height

$G$ _ hole above the neutral axis

$\mathrm{D}$ - hole below the neutral axis

Ac - combined glulam beam

$r$ - radius of curvature for rectangular opening 

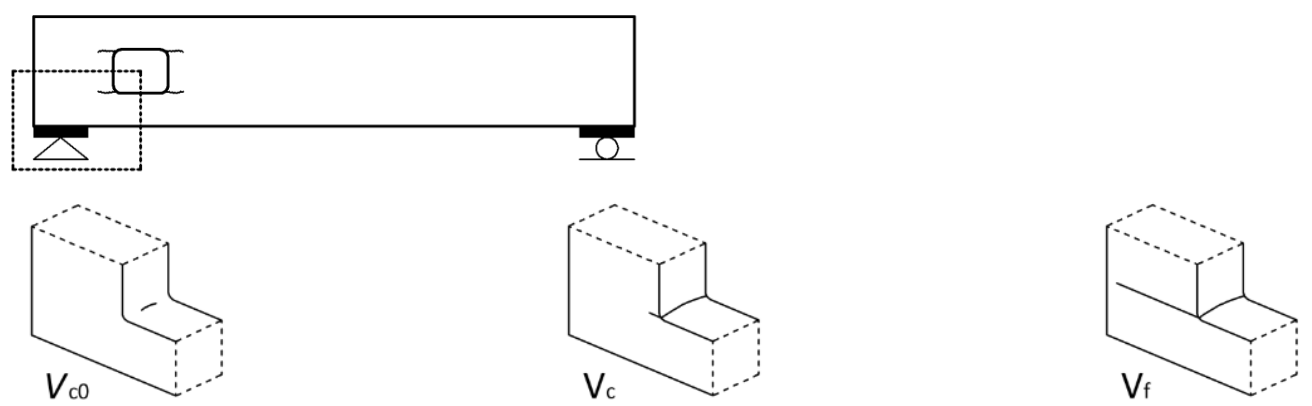

Figure 8 Notations for transverse forces and illustration of crack propagation [19]

Table 3 gives the results for beam with opening in the pure bending zone, showing three characteristic values of the bending moment: $M_{\mathrm{c}, 0}, M_{\mathrm{c}}$, and $M_{\mathrm{f}}$. The subscripts of these variables have the same meanings as those for the transverse forces. Because of the better overview and comparison of results, only the mean values and calculated standard deviations are given.

Table 3 Results for beams with openings in the pure bending zone

\begin{tabular}{|c|c|c|c|c|c|c|c|c|c|c|}
\hline $\begin{array}{l}\text { Researcher } \\
\text { (year) }\end{array}$ & TS & $\begin{array}{l}T \times H \\
{[\mathrm{~mm}]}\end{array}$ & $\begin{array}{c}\phi \\
a \times b \\
{[\mathrm{~mm}]}\end{array}$ & $r$ & $\begin{array}{l}\frac{M}{V H} \\
{[-]}\end{array}$ & $n$ & $\begin{array}{l}M_{\mathrm{c}, 0} \\
\text { mean std } \\
{[\mathrm{kNm}]}\end{array}$ & $\begin{array}{l}M_{\mathrm{c}} \text {, } \\
\text { mean std } \\
{[\mathrm{kNm}]}\end{array}$ & $\underset{\text { mean }}{M_{f}}$ std & LoC \\
\hline $\begin{array}{l}\text { Kolb and } \\
\text { Frech } \\
\text { (1977) [11] }\end{array}$ & $\begin{array}{l}B \\
B\end{array}$ & $\begin{array}{l}80 \times 550 \\
80 \times 550\end{array}$ & $\begin{array}{c}300 \\
300 \times 300\end{array}$ & $?$ & $\begin{array}{l}\infty \\
\infty\end{array}$ & $\begin{array}{l}2 \\
2\end{array}$ & & & $\begin{array}{ll}140.0 & 0.0 \\
136.8 & 4.5\end{array}$ & $\begin{array}{l}\mathrm{m} \\
\mathrm{m}\end{array}$ \\
\hline $\begin{array}{l}\text { Johannesson } \\
\text { (1983) [13] }\end{array}$ & $\begin{array}{l}B \\
B \\
B\end{array}$ & $\begin{array}{l}90 \times 500 \\
90 \times 500 \\
90 \times 500\end{array}$ & $\begin{array}{c}250 \\
1110 \times 370 \\
396\end{array}$ & 25 & $\begin{array}{l}\infty \\
\infty \\
\infty\end{array}$ & $\begin{array}{l}1 \\
1 \\
1\end{array}$ & & $\begin{array}{l}114.0 \\
38.6 \\
50.0\end{array}$ & 122.7 & $\begin{array}{l}\text { It, rt } \\
\text { It, rt } \\
\text { It, rt }\end{array}$ \\
\hline
\end{tabular}

\section{REVIEW OF STANDARDS AND REGULATIONS}

The method for determining the bearing capacity of glulam beams with openings is treated differently in various standards for timber design. Even different editions of the same standards have changed their recommendations and rules for this problem. For example, the European timber design code EN 1995:2004 [2] does not discuss glulam beams with openings, while in a previous version prEN 1995-1-1: Final Draft [21] it provided such guidelines and recommendations based on an analogy with notched beams. These procedures are a modified version of Swedish regulations for timber structures (Limtrahandbok) [22]. The Swedish regulations proposed two methods, one based on empirical expressions, and the other based on an analogy with notched beams. The German timber design code DIN 1052:2004-08 [23] gave rules and guidelines for designing glulam beam with openings and no reinforcement, but in 2007 it withdrew its recommendations for safety reasons. In a later version DIN 1052:2008-12 [24] (and DIN EN 1995-1-1/NA: 2010 [25]) it included new, stricter recommendations for unreinforced beams and for reinforced openings. This standard defines a minimum opening diameter of $50 \mathrm{~mm}$, allowing for smaller diameters only with reduced cross-sectional areas. It does not require reinforcement around the openings for serviceability classes I and II, but reinforcement is mandatory for class III. It also includes recommendations for internal reinforcement, such as drilled screws, and external reinforcement over plywood boards and similar materials. Table 4 compares the recommendations of the DIN EN 1995-1-1/NA: 2010 standard and Swedish regulations (Limtrahandbok) for glulam beams with openings; the variables presented in this table are defined graphically in Figure 9. The Swedish regulations allow openings to be placed in the compression and tension zone of the beam, while the DIN recommendation requires them to be placed closer to the neutral axis. The German code is also more conservative in the size and spacing of the openings, especially when they are placed around the support. 
Table 4 Regulations concerning hole geometry and placement

\begin{tabular}{|c|c|c|c|}
\hline & \multirow{2}{*}{ Limtrahandbok [22] } & \multicolumn{2}{|c|}{ DIN EN 1995-1-1/NA:2010 [25] } \\
\hline & & without reinforcement & with reinforcement \\
\hline la & no restrictions & $\geq 0.5 \mathrm{H}$ & $\geq 0.5 \mathrm{H}$ \\
\hline Iv & no restrictions & $\geq H$ & $\geq H$ \\
\hline$I_{z}$ & $\geq H$ & $\geq 1.5 \mathrm{H} ; \mathrm{min} .300 \mathrm{~mm}$ & $\geq H ; \min .300 \mathrm{~mm}$ \\
\hline$h_{u}$ & $\geq 0.15 \mathrm{H}$ & $\geq 0.35 \mathrm{H}$ & $\geq 0.25 \mathrm{H}$ \\
\hline$h_{\text {। }}$ & $\geq 0.15 \mathrm{H}$ & $\geq 0.35 \mathrm{H}$ & $\geq 0.25 \mathrm{H}$ \\
\hline$a$ & $\leq 3 b$ & $\leq 0.4 H$ & $\leq 3 b$ \\
\hline$b$ ili $\varnothing$ & $\leq 0.5 \mathrm{H}$ & $\leq 0.15 H$ & $\left.\left.\leq 0.3 H^{1}\right) ; \leq 0.4 H^{2}\right)$ \\
\hline$r$ & $\geq 25 \mathrm{~mm}$ & $\geq 15 \mathrm{~mm}$ & $\geq 15 \mathrm{~mm}$ \\
\hline
\end{tabular}

1) - with internal reinforcement

2) - with external reinforcement
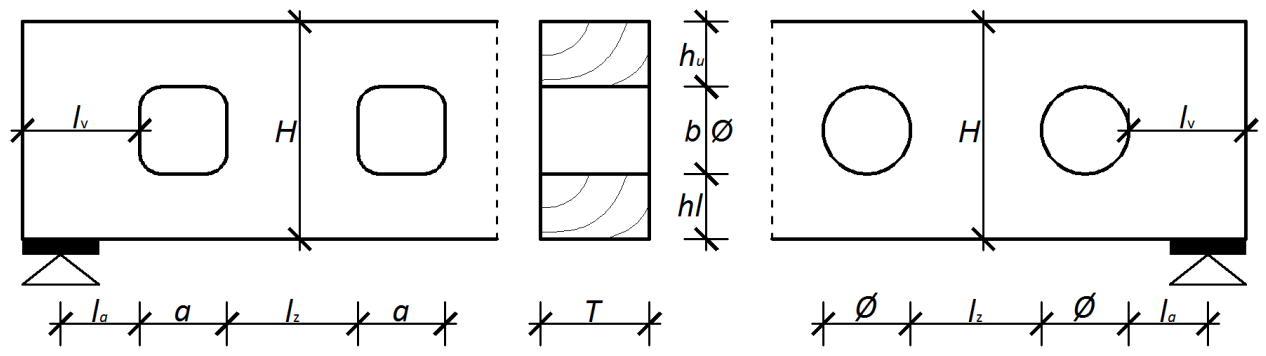

Figure 9 Dimensions of the girder with openings [25]

Table 5 compares the results of experimental testing and design procedures given in the Swedish standards (Limtrahandbok), DIN 1052:2004-08, and prEN 1995-1-1: Final Draft. These experimental results for circular openings were taken from Höfflin [16] and Aicher and Höfflin [17]; for rectangular openings, they were taken from Danielsson [19].

Table 5 Comparison of experimental results with regulations and standards

\begin{tabular}{|c|c|c|c|c|c|c|}
\hline \multirow{2}{*}{$\begin{array}{l}\text { Researcher } \\
\text { (year) }\end{array}$} & \multirow[b]{2}{*}{ Test setup } & \multicolumn{2}{|c|}{ Test results } & \multicolumn{3}{|c|}{ Characteristic value of shear force $V_{i, k}$} \\
\hline & & $\bar{V}_{i}$ & $V_{i, k}$ & $\begin{array}{l}\text { Limtrahandbok } \\
\text { empirical method }\end{array}$ & $\begin{array}{c}\text { Eurocode } 5 \\
\text { prEN 1995-1-1 }\end{array}$ & DIN 1052:2004-08 \\
\hline \multirow{8}{*}{$\begin{array}{l}\text { Höfflin } \\
\text { (2005) [16] }\end{array}$} & $B$ & 106.4 & 79.6 & 83.7 & 176.4 & 116.5 \\
\hline & $B$ & 96.4 & 72.2 & 66.4 & 134.7 & 88.2 \\
\hline & $B$ & 69.2 & 51.8 & 51.9 & 108.0 & 72.8 \\
\hline & $B$ & 55.1 & 41.3 & 66.4 & 134.7 & 63.8 \\
\hline & A & 76.8 & 57.5 & 41.8 & 109.4 & 58.3 \\
\hline & A & 65.5 & 49.0 & 33.2 & 95.8 & 44.1 \\
\hline & A & 47.6 & 35.6 & 25.9 & 77.9 & 36.4 \\
\hline & $A$ & 58.0 & 43.4 & 33.2 & 95.8 & 31.9 \\
\hline \multirow{3}{*}{$\begin{array}{l}\text { Aicher and } \\
\text { Höfflin } \\
\text { (2006) [17] }\end{array}$} & C & 106.4 & 79.6 & 83.7 & 176.4 & 78.1 \\
\hline & C & 61.6 & 46.1 & 51.9 & 108.0 & 54.9 \\
\hline & $\mathrm{C}$ & 48.8 & 36.5 & 25.9 & 77.9 & 27.4 \\
\hline \multirow{9}{*}{$\begin{array}{l}\text { Danielsson } \\
(2008) \text { [19] }\end{array}$} & $A^{S}$ & 57.3 & 50.1 & 36.6 & 60.1 & 41.8 \\
\hline & $A^{S}$ & 53.2 & 46.6 & 30.8 & 50.6 & 37.6 \\
\hline & $A^{G}$ & 55.7 & 48.8 & 36.6 & 53.3 & 35.9 \\
\hline & $A^{D}$ & 50.0 & 43.8 & 36.6 & 53.3 & 35.9 \\
\hline & Fs & 62.2 & 54.5 & 36.6 & 60.1 & 50.2 \\
\hline & $A^{S}$ & 25.6 & 22.4 & 10.5 & 32.1 & 11.9 \\
\hline & $A^{G}$ & 23.4 & 20.5 & 10.5 & 28.5 & 10.2 \\
\hline & $A^{D}$ & 23.0 & 20.2 & 10.5 & 28.5 & 10.2 \\
\hline & $\mathrm{F}^{\mathrm{S}}$ & 26.6 & 23.3 & 10.5 & 32.1 & 14.3 \\
\hline
\end{tabular}


Comparing these results shows that Swedish and German timber design codes underestimate the capacity of all samples with rectangular openings. The underestimation is even greater for smaller samples because these two provisions do not account for the sample size, a parameter which experiments have shown to be important. In later versions of the German standard DIN 1052:2008-12 and DIN EN 1995-1-1/NA: 2010 a factor that takes into account the "size effect" for beams higher than $450 \mathrm{~mm}$ was introduced, but the standard still does not take into account the relative aspect ratio of opening in the expression for resistance. For samples with circular openings, DIN 1052:2004-08 overestimates their capacity because the expression for the crack length gives unrealistically large value and maximum tensile stress perpendicular to the fibers are lower compared to the real situation [26]. The relevant provisions in prEN 1995-1-1: Final Draft are unsafe because they overestimate the beam capacity, compared to all experimental samples. Although the draft standard takes into account the "size effect" as well as the relationship between the section dimensions, it still does not take into account ratio between bending moment and shear force which is also significant. However, this standard can well predict the influence of various parameters. The main reason that German and European timber design code give different approaches for designing glulam beams with openings is because German standard is based on equilibrium of stresses around the opening, while European standard is based on the principles of fracture mechanics.

\section{THEORETICAL METHODS}

Stress analysis in wooden structures has many theoretical backgrounds. Wood is natural and nonhomogeneous, making its properties more complex than steel or concrete. Also, because of its anisotropy, it behaves differently based on many factors, including the stress state. These complexities mean that using simplifications and assumptions in mathematical models might be acceptable for some applications and loads, but for others it might produce incorrect, unsafe results.

Theoretical methods for timber structures can be roughly divided into deterministic and stochastic methods. Deterministic methods treat wood as a homogenous material, while stochastic methods treat it as a heterogeneous material, which is more realistic because wood contains knots, bumps, and other defects. A second way to classify these models is by how they handle the material ductility during failure, distinguishing the stress state that leads to brittle failure those which lead to ductile failure. Table 6 shows the classification of these methods.

Table 6 Models used to analyze the engineering strength of timber [27]

\begin{tabular}{|c|c|c|}
\hline Analysis model & $\begin{array}{c}\text { Deterministic methods } \\
\text { (homogeneous properties) }\end{array}$ & $\begin{array}{c}\text { Stochastic methods } \\
\text { (heterogeneous properties) }\end{array}$ \\
\hline \hline Brittle behavior & Conventional stress analysis & Weibull weakest link theory \\
\hline \multirow{3}{*}{ Ductile behavior } & Linear elastic fracture mechanics & Probabilistic linear elastic fracture mechanics \\
\cline { 2 - 3 } & $\begin{array}{c}\text { Generalized linear elastic fracture } \\
\text { mechanics }\end{array}$ & $\begin{array}{c}\text { Probabilistic generalized linear elastic fracture } \\
\text { mechanics }\end{array}$ \\
\cline { 2 - 3 } & Nonlinear fracture mechanics & Probabilistic nonlinear fracture mechanics \\
\hline
\end{tabular}

The most used calculation method for designing timber structures is conventional stress analysis where the wood is considered as homogeneous and linear elastic material. However, this simplification often leads to incorrect results in many applications, among them for the design of beams with openings.

Until the 1990s, most theoretical approaches used conventional stress analysis, where a material is exposed to a complex stress state. Significant research in this era was performed by Kolb and Frech [11], Penttala [12], and Johannesson [13]. Linear elastic fracture mechanics is used for the crack propagation analysis. The method is based on the assumption of an ideal linear elastic material behavior and the presence of crack or sharp notch. The method can not determine the expected position of crack but only will the existing crack develop or not. The method involves three sub methods where the first is based on an energy approach, the other one on the stress intensity factor and the third one on the J-integral method. Generalized linear elastic fracture mechanics includes two sub methods: a method of average stress and method of initial crack. First sub method is based on calculation of mean stresses in the area of crack opening and their insertion in conventional stress analysis equations. From the 1990s onward, most theoretical approaches used the assumptions of fracture mechanics. Significant research in this era was performed by Pizio [14], Hallström [15, 28], Riipola [29, 30], 
Aicher, Schmidt, and Brunhold [31], Petersson [32], Gustafsson and Serrano [33], Scheer and Hasse [34], and Stefansson [35]. Aicher et al. [5] implemented 2D FEM analysis using orthotropic material and 3D FEM analysis with a cylindrically anisotropic material. Each particular lamina around the opening was modeled in the function of the orientation of its growth rings. Modeling lamella with cylindrically anisotropic material resulted with agreement with experiment. Aicher and Hofflin [9] have proposed a design method which is based on a probabilistic approach to determine capacity where material is modeled as heterogeneous. The aim of these method is to express the degree of heterogeneity in a way that real complex heterogeneous stress state around the hole is converted in the equivalent homogeneous state which has an equal probability of failure. Aicher and Hofflin concluded that the method gives good results for value of transverse force at the time of the opening of cracks along the entire width of beam $V_{c}$ while in case of transverse force at failure $V_{f}$ is not applicable. Also, the disadvantage of this method is that it can not be applied on singularity problem of stresses caused by sharp crack or notch.

Danielsson and Gustafsson [20] applied the probabilistic method of fracture mechanics to analyze rectangular openings in glulam beams. Gustafsson and Serrano [33] proposed a new method that generalizes linear elastic fracture mechanics, combining Weibull theory and the average stress method. Combining these two methods, they accounted for energy through fracture mechanic and for stochastic material characteristics through Weibull theory. Their method predicted material strength very well except in small samples, where it overestimated the bearing capacity. The reason for this is too long potential breakdown area around the hole, which finally leads to reduced peak stress and thereby increased load capacity. The same deficiency is observed in German standard DIN EN 1995-1-1/NA: 2010.

\section{CONCLUSION}

Ultimately, the experimental and theoretical work described here are useful for creating rules and design regulations that are simple, generalizable, and practical. However, glulam beams are complicated to design, and the various theoretical approaches for these beams give very different results. Additionally, many parameters that influence these beams have not yet been examined. Because of these complexities and uncertainties, leading regulations for timber structures have completely specifications for omitted glulam beams with openings, leaving space for further research.

Because experiments on downscaled samples have doubtful reliability, more experiments on beams with realistic dimensions are necessary, especially for beams with large spans (over $8 \mathrm{~m}$ ), which have little experimental data. Additionally, there is little experimental data on girders with large ratios between height and width, whose behavior would be more similar to shear walls than to beam girders, and whose impact on stability would be important in determining the failure mechanism. Also, there is little data on beams with variable crosssections, including trapezoidal beams (single- and double-tapered) in which a complex stress state already exists.

Experimental studies have generally kept the section width constant, so experiments are needed to determine how it contributes to capacity. Also, studies have mostly kept applied loads to short durations, so experiments are needed that expose beams to long-term loads, studying the additive effects of creep and moisture changes. Finally, studies have mostly examined simply supported beams, but real structures are often statically indeterminate, containing additional stresses whose influences are unknown.

\section{References}

[1] Danielsson H. 2007: The strength of glulam beams with holes - A survey of tests and calculation methods, Report TVSM-3068, Division of Structural Mechanics, LTH, Lund University.

[2] Eurocode 5: Design of timber structures - Part 1-1: General - Common rules and rules for buildings EN 1995-1-1:2004 (E).

[3] Aicher S.; Höfflin L. 2004: New design model for round holes in glulam beams, Proceedings of 8th World Conference on Timber Engineering, Vol. 1, pp. 67 - 72, Lahti, Finland.

[4] Aicher S.; Höfflin L. 2000: A contribution to the analysis of glulam beams with round holes, Otto-GrafJournal, Volume 11, pp. 167 - 180. 
[5] Aicher S.; Höfflin L.; Reinhardt H. 2003: Verifizierung versagenrelevanter Dehnungsverteilungen im Bereich runder Durchbruche in Brettschichtholztragern, Bautechnik, Volume 80, No. 8, pp. 523 - 533.

[6] Aicher S.; Höfflin L. 2001. Runde Durchbrucke in Biegetragern aus Brettschichtholz, Teil 1: Berechnung, Bautechnic, Volume 78, No. 10, pp. 706 - 715.

[7] Aicher S.; Höfflin L. 2003: Design of rectangular holes in glulam beams, Otto-Graf-Journal, Volume 14, pp. $211-229$

[8] Aicher S.; Zisi N. 2013: Design of large rectangular holes in compression and moment loaded glulam members, Pro ligno, Volume 4, No. 4, pp. 50 - 59.

[9] Aicher S.; Höfflin L. 2008: Fracture behavior and design of glulam beams with round holes, Proceedings of 10th World Conference on Timber Engineering, Volume 1, pp. 132-140, Miyazaki, Japan.

[10] Bengtsson S, Dahl G. 1971: Influence of holes near support on the strength of glulam beams (In Swedish), Master's Thesis, Byggnadsteknik II, LTH, Lund University.

[11] Kolb H.; Frech P. 1977: Untersuchungen an durchbrochenen bindern aus brettschichtholz, Holz als Roh und Werkstoff 35, pp. 125-134 (in German).

[12] Penttala V. 1980: Glulam beams with holes, Publication 33, Division of Structural Engineering, University of Technology Otaniemi, Helsinki.

[13] Johannesson B. 1983.: Design problems for glulam beams with holes, Dissertation, Division of Steel and Timber Structures, Chalmers University of Techology, Göteborg.

[14] Pizio S. 1991: Die Anwendung der Bruchmechanik zur Bemessung von Holzbauteilen, untersucht am durchbrochen und am ausgeklinkten Träger (in German), Dissertation, Baustatik und Stahlbau, ETH, Zürich, Switzerland.

[15] Hallström S. 1995: Glass fibre reinforced laminated timber beams with holes. Report 95-12, Department of Lightweight Structures, Royal Institute of Technology (KTH), Stockholm.

[16] Höfflin L. 2005: Runde Durchbrücke in Brettschichtholzträger - Experimentelle und theoretische Untersuchungen, Ph.D Thesis, MPA Otto-Graf-Institute, University of Stuttgart (in German).

[17] Aicher S.; Höfflin L. 2006: Tragfähigkeit und Bemessung von Brettschichtholzträgern mit runden Durchbrüchen - Sicherheitsrelevante Modifikationen der Bemessungsverfaren nach Eurocode 5 und DIN 1052 MPA Otto-Graf-Institute, University of Stuttgart (in German).

[18] Danielsson H. 2008: Strength tests of glulam beams with quadratic holes - Test report, Report TVSM-3068, Division of structural mechanics, Lund University.

[19] Danielsson H.; Gustafsson P.J. 2008: Strength of glulam beams with holes - Tests of quadratic holes and literature test result compilation, CIB-W18/41-12-4, St. Andrews, Canada.

[20] Danielsson H.; Gustafsson P.J. 2010: A probabilistic fracture mechanics method and strength analysis of glulam beams with holes, European Journal of Wood and Wood Products, Volume 69, Issue 3, pp. 407-419

[21] Eurocode 5: Design of timber structures - Part 1-1: General Rules - General rules and rules for buildings prEN 1995-1-1: Final Draft, 2002-10-09.

[22] Carling O. 2001: Limtrahandbok (glulam handbook), Svenskt Limtra AB, Print \& Media Center i Sundsvall $A B$, Stockholm, Sweden.

[23] DIN 1052: 2004-08: Design of timber structures - General rules and rules for buildings (in German).

[24] DIN 1052: 2008-12: Design of timber structures - General rules and rules for buildings (in German).

[25] DIN EN 1995 -1-1/NA: 2010. National Annex - Nationally determined parameters - Eurocode 5: Design of timber structures - Part 1-1: General - Common rules and rules for buildings (in German).

[26] Aicher S.; Höfflin L. 2002: Glulam beams with round holes - a comparison of different design approaches vs. test data, CIB-W18/35-12-1, Kyoto, Japan

[27] Danielsson H. 2013: Perpendicular to grain fracture analysis of wooden structural elements, Ph.D thesis, Division of Structural Mechanics, Lund University.

[28] Hallström S. 1995: Glass fibre reinforcement around holes in laminated timber beams. Report 95-14, Department of Lightweight Structures, Royal Institute of Technology (KTH), Stockholm.

[29] Riipola K. 1995: Timber beams with holes. Fracture mechanics approach. Journal of Structural Engineering, Volume 121, Issue 2, pp. 225-239.

[30] Riipola K. 1995: Design of glulam beams with holes, CIB-W18/28-12-3, Copenhagen, Denmark

[31] Aicher S.; Schmidt J.; Brunhold S. 1995: Design of timber beams with holes by means of fracture mechanics, CIB-W18/28-19-4, Copenhagen, Denmark. 
[32] Petersson H. 1995: Fracture design analysis of wooden beams with holes and notches. Finite element analysis based on energy release rate approach, CIB-W18/28-19-3, Copenhagen, Denmark.

[33] Gustafsson P.J., Serrano E. 1999: Fracture Mechanics in timber engineering - Some methods and applications, Proceedings of 1st RILEM Symposium on Timber Engineering, p. 141-150, Stockholm, Sweden

[34] Scheer C.; Haase K. 2000: Durchbrüche in Brettschichtholztrager, Teil 1: Spannungstheoretische Untersuchungen, Holz als Roh - und Werkstoff, Volume 58, pp. 153-161 (in German).

[35] Stefansson F. 2001: Fracture analysis of orthotropic beams - Linear elastic and nonlinear methods. Report TVSM-3029, Division of Structural Mechanics, LTF, Lund University 